Portland State University

PDXScholar

6-19-2018

\title{
Molecular Probes Selectively Targeting Pancreatic Cancer for Imaging
}

Brian Viet Truong

Portland State University

Follow this and additional works at: https://pdxscholar.library.pdx.edu/honorstheses Let us know how access to this document benefits you.

\section{Recommended Citation}

Truong, Brian Viet, "Molecular Probes Selectively Targeting Pancreatic Cancer for Imaging" (2018). University Honors Theses. Paper 629.

https://doi.org/10.15760/honors.640

This Thesis is brought to you for free and open access. It has been accepted for inclusion in University Honors Theses by an authorized administrator of PDXScholar. Please contact us if we can make this document more accessible: pdxscholar@pdx.edu. 
Brian Truong

Thesis

Molecular Probes Selectively Targeting Pancreatic Cancer for Imaging

\section{Abstract:}

Hypoxia is a condition where oxygen is unable to reach tissues and cells. When these areas become oxygen deprived, they are unable to synthesize reactive oxygen species (ROS), in whose absence, specific enzymes become overexpressed. Nitroreductase is a protein commonly found in areas where hypoxic tumors are located and also where many cancer forms occur. Nitroreductase is a good indicator of hypoxic tumors and can be detected by molecular probes. One of the few synthesized probes termed compound 12 is highly tumor specific and enable visualization of pancreatic cancer at four different levels of structure. Compound $\mathbf{1 2}$ works by fluorescing in pancreatic cancer cell, indicating regions where metastasis and abnormal development is occurring. We are also synthesizing a derivative of compound 12 by adding a nitro(-NO2) group.

Derivative compound $\mathbf{1 2}$ has a strong potential to enhance the visualization of not only pancreatic cancer, but also cancer throughout the body and provide, therapeutic monitoring, and aid to surgeons in margin assessment.

\section{Background Information:}

Pancreatic cancer affects more than 53,000 people in the United States. Scientist and researchers estimate that pancreatic cancer may soon be the second leading cause of disease-related death by 2030 (Wang, 2017). Also, what makes pancreatic cancer so 
dangerous is that people have a post-diagnosis life expectancy of 5-7 months. Pancreatic cancer is correlated with hypoxic environments and the overexpression of enzymes like nitroreductase.

Hypoxic tumor cells are deprived of oxygen. In cancer patients, as the tumor grows, the mass begins to outgrow its blood supply, leaving some regions with oxygen concentrations lower than average. This inhibits the synthesis of enzymes and coenzymes for metabolic function. Hypoxia can be harmful to both cancer cells and healthy cells undergoing genetic and adaptive changes that increases survival and growth rate (Harris, 2002).

It is also important to note the physiological and morphological changes in the vasculature of these areas. Typically, in cancer cells, and especially pancreatic cancer, they are growing and expanding the tumors, the oxygen supply will surpass oxygen demand and eventually the distance between cells and vasculature increases, complicating oxygen diffusion and creating an even more hypoxic environment (Muz, 2015). This can be locally detrimental and can spread, infecting other parts of the body. Also, hypoxic tumor cells have a higher resistance towards cancer treatments and are more likely to metastasize (Guo, 2013).

Nitroreductase, which is commonly overexpressed where oxygen is in low abundance, is a crucial protein. It helps cells reduce nitrogen compounds. What makes nitroreductase a vital enzyme to understand is that it's generally found in hypoxic cells 
where they can flow in blood vessels, making it difficult to target with selective drugs (Denny, 2000). This leads to cells becoming resistant to chemotherapy and radiation. By using molecular probes, one can select regions high in nitroreductase accurately. An example of a molecular probe is determined by compound 12. Compound $\mathbf{1 2}$ has highly attractive characteristics such as high contrast, and pancreas tumor specificity (Wang, 2017). With these regions highly fluorescent, they can potentially provide surgeons a guided surgery through live imaging and enhance the detection of pancreatic cancer (Wang, 2017).

\section{Methods:}

Molecular probes can be challenging to design, synthesize and evaluate. Examples of potential properties can include reacting to different hypoxic environments and various levels of structure. While compound $\mathbf{1 2}$ selectively target pancreatic cancer cells, the derivative compound 12 was synthesized to target other regions that could be expressing forms of cancer.

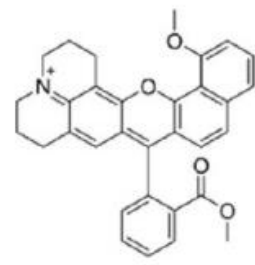

12
$\mathrm{MW}=490.57 \mathrm{~g} / \mathrm{mol}$

$\log D=1.91$

Formal charge $=+1$

$\mathrm{H}$-bond donors/acceptors $=0 / 4$

Polar surface area $=47.77 \AA^{2}$

Dipole moment $=6.52$ Debye

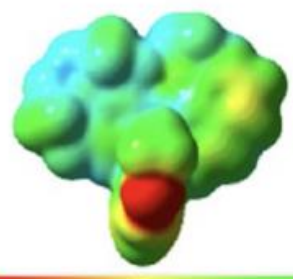

0.05

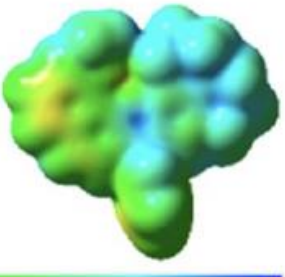

0.12

Figure 1. The chemical structures and physiochemical properties of compound 12 (Wang, 2017)

To synthesize both the probes, a variety of techniques were involved that began with the condensation of hydroxybenzophenones with naphthols in a mixture of 
$\mathrm{CH} 3 \mathrm{SO} 3 \mathrm{H} / \mathrm{TFA}$ in a $1: 1$ ratio. A temperature of $80^{\circ} \mathrm{C}$ was maintained for $16-24$ hours to produce lactones or carboxylates. After this was accomplished, we followed with fischer esterifications to produce methyl derivatives, which was then carried out my methanol and catalyzed by either hydrochloric acid or sulfuric acid. We further the process by treating either the carboxylate or methyl ester derivative with methyl iodide while in the presence of potassium carbonate in dimethylformamide to produce the methyl ethers. The base structure for both the compound should be exactly the same, the only difference is in the end. For compound 12, we attached a methoxy group towards the end, where as in the derivate compound $\mathbf{1 2}$, we attached a nitro group instead. Lastly, the compounds were purified and isolated by column chromatography along with the characterization through NMR and mass spectrometry.

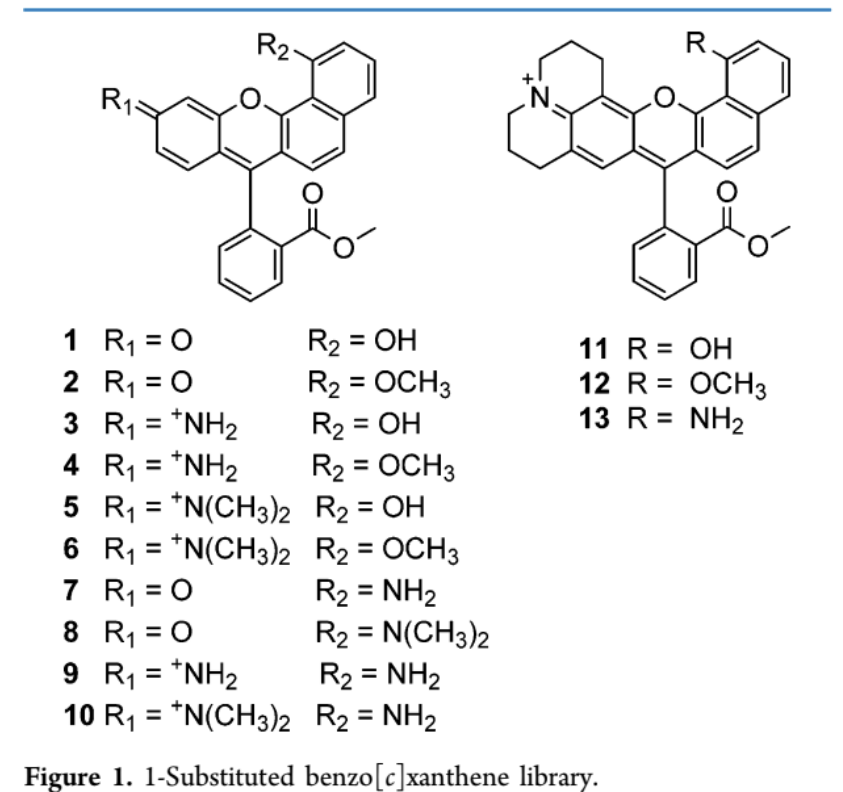

Figure 2. Compounds that were involved in the synthesis and the modifications had to be done in a benzene substitution (Wang, 2017). 
The compounds were analyzed using NMR or nuclear magnetic resonance spectroscopy. The NMR spectrometers help identify the types of compounds present in the mixture and if there are impurities. To use the NMR spectrometer, a small sample of our product is collected in a tube and placed in a giant magnet. The results are displayed on a computer screen and will show the different resonance(peaks) associated with the various compounds to enable assignment of the product structure.

Fluorescence Spectroscopy and Ultraviolet-Visible Spectroscopy are used to determine the optical properties of the fluorophores. Fluorescence Spectroscopy analyzes the fluorescence emitted from the sample. Ultraviolet-Visible Spectroscopy examines the absorption of light by the sample. Typically, Ultraviolet-Visible Spectroscopy and fluorescence spectroscopy are used together in analyzing the compounds.

Fluorescence spectroscopy measures the transitions from the excited state to ground state; whereas in Ultraviolet-Visible Spectroscopy, it measures the changes from ground state to excited state. 


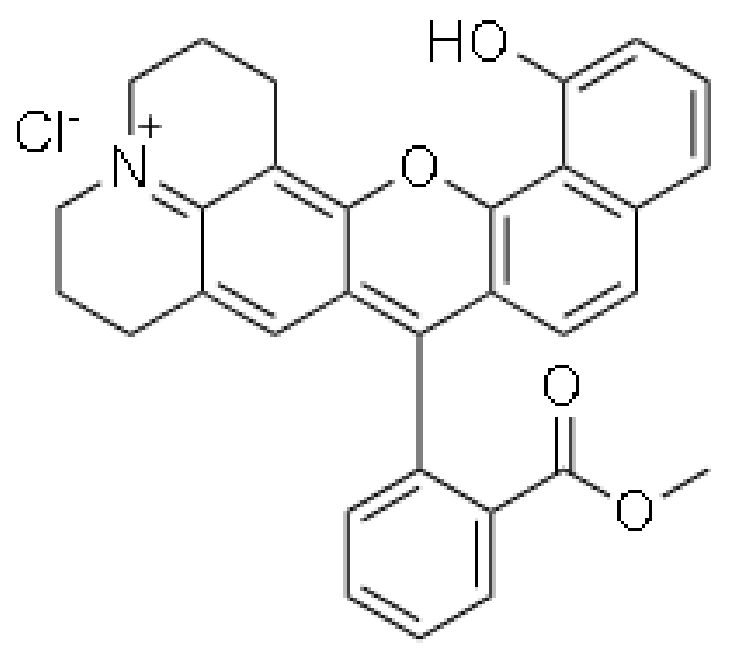

Compound 12

Figure 3. This figure shows the final structure obtained once the reaction had been completed.

\section{Compound 12}

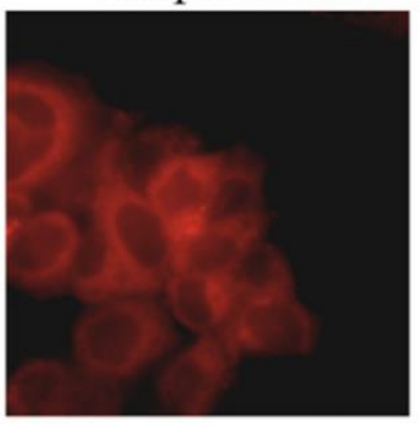

Merge

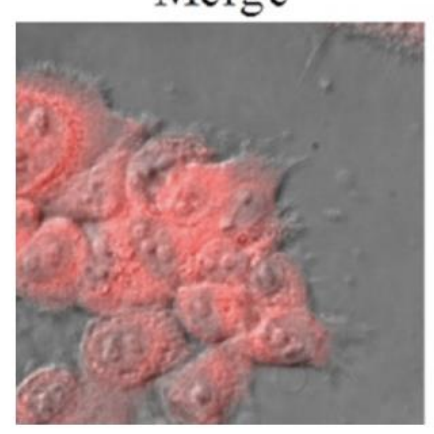


Figure 4. The fluoresce emission of compound $\mathbf{1 2}$ in the healthy mouse cell line (Wang, 2017).

$\underline{\text { Results: }}$

Compound 12 was tested on a mouse Pancreatic Ductal Adenocarcinoma (PDAC) cell line known as Capan-1. The molecular probe showed high selectivity to fluoresce in the mitochondria and also slightly in the nucleus and endoplasmic reticulum. The reason why compounds 12 fluoresce in the mitochondrial environment is that the structure is considered cationic, and thus distribute in the mitochondrial matrix due to the electric potential across the mitochondrial membrane (Wang, 2017).

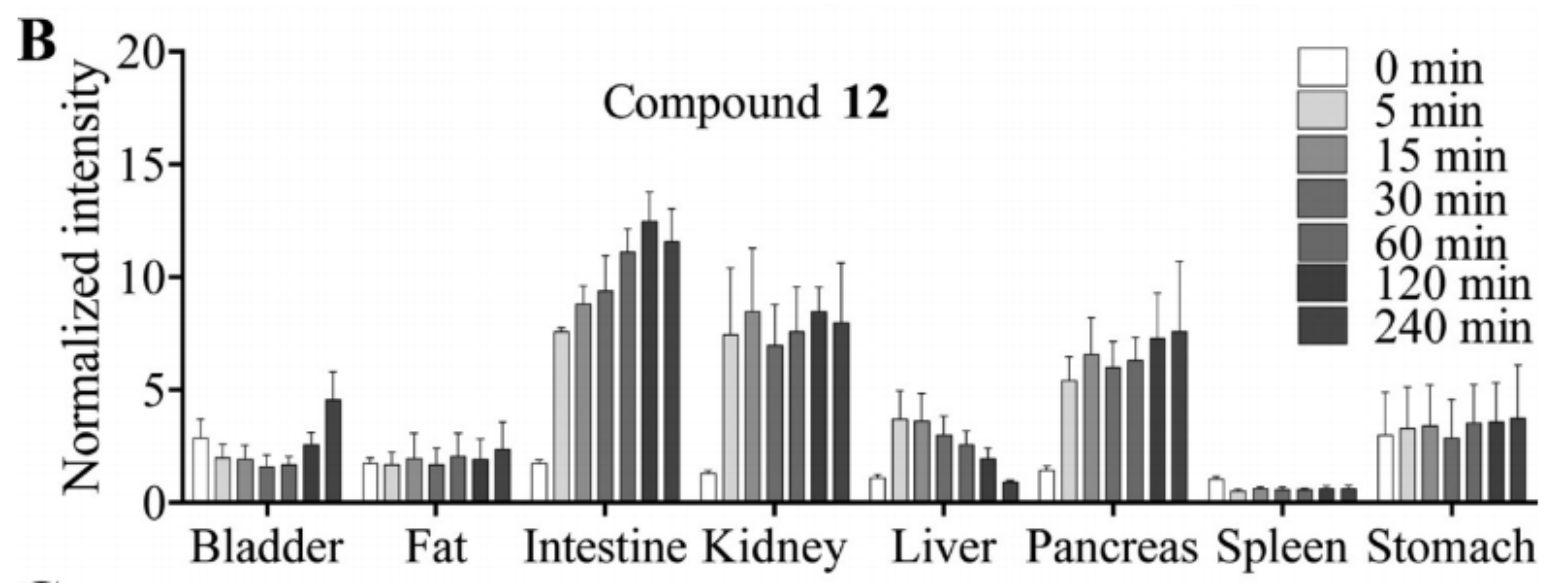

Figure 5. This figure represents the intensity of each fluorescent probes in various organs of a healthy mouse (Wang, 2017). 
The compound 12 was tested on mice with an oncogenic mutation termed KRAS. The mice that expressed KRAS developed similar symptoms to Pancreatic Ductal Adenocarcinoma seen in human cancer patients. The approval for the use of such animals was obtained by The Institutional Animals Care and Use Committee (IACUC) at OHSU. Microscopic images provide a real-time comparison between the molecular probes and color. What we found was that the compound 12, displayed a high selectively for these cancerous tissues seen in pancreatic cancer. While the compound 12 shows high selectivity for regions expressing pancreatic cancer, the derivative of compound $\mathbf{1 2}$ is still in process of testing and analyzing.

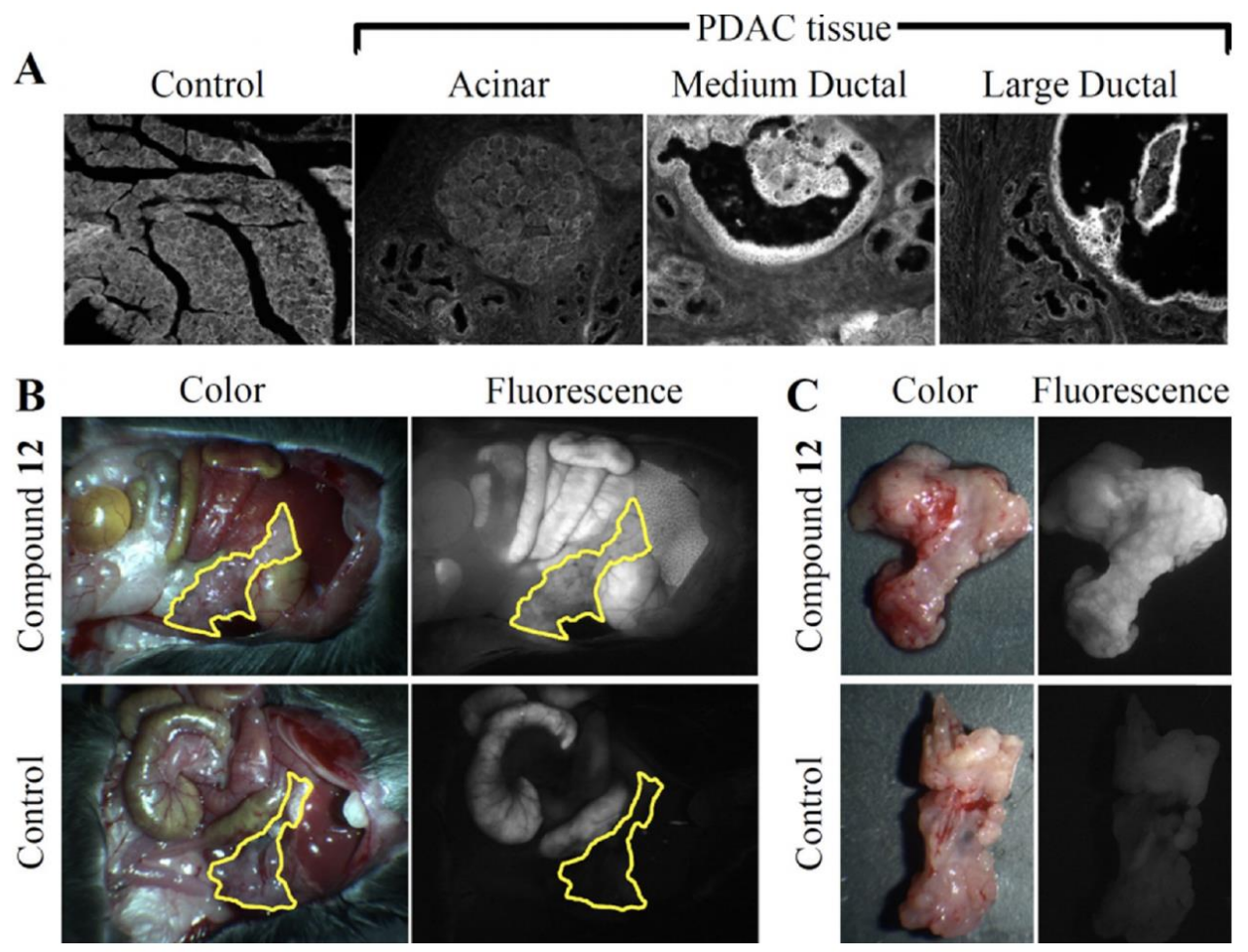

Figure 6. These images represent microscopic images of compound 12 stained on pancreatic tissues (Wang, 2017). 


\section{Discussion:}

The synthesis of the compound $\mathbf{1 2}$ provides insightful knowledge on the imaging of pancreatic cancer through molecular probes. Compound 12 provides high resolution and selectivity in cancerous tissues, identifying distinctions between individual cell morphologies. This project is vital to the understanding of pancreatic cancer and how these areas can be determined. These probes can serve in the medical community for imaging disease-relevant cancer. Also, clinicians and researchers can apply these probes to biomedical research to better understand the development of tumors. This will allow for more accurate treatment plans and an accurate perspective on the past of the tumors. Overall, compound $\mathbf{1 2}$ is pancreas tumor-specific and can help the medical community, promoting enhanced pancreatic cancer detection, therapeutic monitoring, and research.

\section{Bibliography:}

Cui, L., Zhong, Y., Zhu, W., Xu, Y., Du, Q., Wang, X., ... \& Xiao, Y. (2011). A new prodrugderived ratiometric fluorescent probe for hypoxia: high selectivity of nitroreductase and imaging in tumor cell. Organic letters, 13(5), 928-931.

Denny, W. A. (2000). The role of hypoxia-activated prodrugs in cancer therapy. The lancet oncology, 1(1), 25-29. 
Muz, B., de la Puente, P., Azab, F., \& Azab, A. K. (2015). The role of hypoxia in cancer progression, angiogenesis, metastasis, and resistance to therapy. Hypoxia, 3, 83.

Harris, A. L. (2002). Hypoxia-a key regulatory factor in tumour growth. Nature Reviews Cancer, 2(1), 38.

Xu, J., Sun, S., Li, Q., Yue, Y., Li, Y., \& Shao, S. (2015). A rapid response "Turn-On" fluorescent probe for nitroreductase detection and its application in hypoxic tumor cell imaging. Analyst, 140(2), 574-581.

Li, Z., Li, X., Gao, X., Zhang, Y., Shi, W., \& Ma, H. (2013). Nitroreductase Detection and Hypoxic Tumor Cell Imaging by a Designed Sensitive and Selective Fluorescent Probe, 7-[(5-Nitrofuran2-yl) methoxy]-3 H-phenoxazin-3-one. Analytical chemistry, 85(8), 3926-3932.

Wang, L., Barth, C. W., Sibrian-Vazquez, M., Escobedo, J. O., Lowry, M., Muschler, J., ... \& Strongin, R. M. (2017). Far-Red and Near-Infrared Seminaphthofluorophores for Targeted Pancreatic Cancer Imaging. ACS omega, 2(1), 154-163.

Guo, T., Cui, L., Shen, J., Zhu, W., Xu, Y., \& Qian, X. (2013). A highly sensitive long-wavelength fluorescence probe for nitroreductase and hypoxia: selective detection and quantification. Chemical Communications, 49(92), 10820-10822. 
Brown, J. M., \& Wilson, W. R. (2004). Exploiting tumour hypoxia in cancer treatment. Nature Reviews Cancer, 4(6), 437.

Logsdon, C. D., Simeone, D. M., Binkley, C., Arumugam, T., Greenson, J. K., Giordano, T. J., ... \& Hanash, S. (2003). Molecular profiling of pancreatic adenocarcinoma and chronic pancreatitis identifies multiple genes differentially regulated in pancreatic cancer. Cancer research, 63(10), 2649-2657. 